\title{
Study on Structural and Dielectrical Properties of some Chloroquinoxaline Derivatives
}

\author{
N. A. EL-Ghamaz ${ }^{1}$, E. A. Gaml ${ }^{1}$, Hajar Abusnina*1 \\ ${ }^{1}$ Department of Physics, Faculty of Science, Damietta University 34517, Egypt. \\ Received: 3 November 2020 /Accepted: 2 December 2020 \\ * Corresponding author's E-mail: hajer.gege2014@gmail.com
}

\begin{abstract}
The structural, thermal and electrical properties of three chloroquinoxaline derivatives are investigated by X-ray diffraction (XRD) and differential scanning calorimetric (DSC) techniques. It is found that the chemical composition affected the structure and thermal stability of each derivative. The dielectric properties of the three derivatives are investigated in the frequency range $100 \mathrm{~Hz}-$ $5 \mathrm{MHz}$ and in the temperature range $300-470 \mathrm{~K}$. The behaviors of the real and imaginary parts of the dielectric constant is investigated. Also, the AC conductivity as a function of both temperature and frequency is studied. The charge carrier's conduction mechanism is found to follow the quantum mechanical tunneling (QMT) model. The complex bulk modulus is also investigated.
\end{abstract}

Keywords: Dielectric properties; Conductivity; Bulk modulus.

\section{Introduction}

The organic substances are classified as polymer, monomer and organic compounds. The investigation of these classes of materials has created completely new scientific concepts and potential for their application of perspective in molecular electronics. They mostly contained an extended $\pi$-electron system which can be transformed from a semiconducting state to a conducting state [1]. As active components in electronics and optoelectronics applications, they are of increasing interest. Since they are versatile, low cost and ease of production, they represent a valid alternative to conventional inorganic semiconductor technology in a number of applications, such as flat panel displays, organic field effect transistors, photovoltaic cells, illumination, plastic integrated circuits and organic light-emitting diodes. Although initial commercial applications of this technology are now being realized, a deeper scientific understanding is still needed in order to achieve optimum performance of the device [2]. Owing to its uses in many technical applications. Organic compounds have gained particular attention such as: molecular switch, temperature indicator devices, temperature sensitive light filters, imaging systems and storage of energy. In this present work we introduce some chloroquinoxaline compounds which contain heterocyclic compounds with benzene ring and a pyrazine ring. Quinoxaline 1;4 dioxide provides number of applications in biology [3] and electronics [4]. It was found that the presence of functional groups and different substituted groups on the quinoxaline derivatives affected their physical properties [5]. Quinoxaline and its derivatives showed 
non-linear optical behavior and can be applied in optical devices such as solar cells [6]. Quinoxaline derivatives are also thermally stable, as they begin to decompose at $200{ }^{\circ} \mathrm{C}$ [7]. In this study, the structural, and electrical properties of three newly synthetized quinoxaline derivatives in bulk form are presented. To the best of our knowledge the structural and electrical properties of these derivatives have not been investigated yet.

\section{Experimental}

\section{Materials}

The compounds under investigation in present work are three heterocyclic Chloroquinoxaline compounds namely: 8-Chloro-1-oxo-2-phenyl1,2-dihydropyrido[3,4-b]quinoxaline $\quad 5,10$ dioxide (1), (E)-6-Chloro-3-(2-(4(dimethylamino)benzylidene)hydrazine-1carbonyl)-2-methylquinoxaline 1,4-dioxide (2) and (E)-6-Chloro-2-(3-nitrostyryl) quinoxaline 1,4-dioxide (3). These compounds have been synthesized previously in the laboratory of the Department of Chemistry, Faculty of Science, Damietta University by Samy B. Said et al. [8]. The chemical composition of these compounds is shown in Fig. 1.
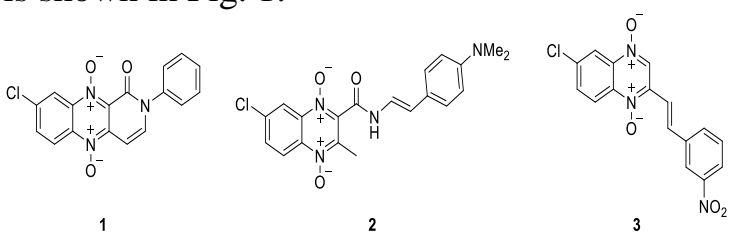

Fig.1: The molecular structural of compounds 1,2 , and3

\section{Measurements and Characterizations}

\section{$X$-ray diffraction $(X R D)$ measurements}

XRD patterns of the investigated powder samples are determined by X-ray diffractometer (Shimadzu XRD 6000). XRD measurements were performed at a scan rate of 2 degrees per second with $\mathrm{CuK} \alpha$ radiation source (wavelength of $0.15406 \mathrm{~nm}$ ), generator voltage of $40 \mathrm{kV}$, generator current of $30 \mathrm{~mA}$ at room temperature.

\section{Differential scanning calorimetric (DSC).}

DSC is a thermal analytical technique in which the difference in the amount of heat required to increase the sample's temperature and the reference is measured as a function of temperature. DSC measurements are performed in the temperature range $300-673 \mathrm{~K}$ using Shimadzu DSC-50 in nitrogen atmosphere at a flow rate of $10 \mathrm{~mL} / \mathrm{min}$ using the aluminum cell and a temperature increase rate of $10^{\circ} \mathrm{C} /$ min.

\section{AC Electrical measurements.}

The powder of samples 1, 2, and 3 is compressed by a hydraulic press under a pressure of 10 tons $/ \mathrm{cm}^{2}$ at room temperature to form pellets. The thickness and surface area of each the pellet is about $\left(0.5 \mathrm{~mm}\right.$ and $\left.21 \mathrm{~mm}^{2}\right)$, $\left(0.58 \mathrm{~mm}\right.$ and $\left.25 \mathrm{~mm}^{2}\right)$ and $(23 \mathrm{~mm}$ and 20 $\mathrm{mm}^{2}$ ) for samples 1,2 and 3 , respectively. Silver paste is used to make Ohmic contacts onto the two sides of the pellets. The AC electrical measurements are performed using LCR bridge model 3522-50 HIOKI. The measurements are performed in the frequency range $100 \mathrm{~Hz}-5$ $\mathrm{MHz}$ and in the temperature range $320-460 \mathrm{~K}$.

\section{Results and discussion}

\section{$X$-ray diffraction analysis}

XRD patterns for powder compounds 1,2 and 3 are shown in Fig 2. It is noticed that compounds 1 and 2 demonstrate polycrystalline and amorphous phases due to the presence of many sharp peaks which are accompanied with a broad hump at around $2 \theta \approx 25^{\circ}$. Also it is noticed that compound 3 has no sharp peaks and has only a hump at $2 \theta \approx 25^{\circ}$ Fig 3 . This confirms that this compound has a completely amorphous phase. To determine the crystal structure of compounds 1 and 2, Fullproof software [9] is used to find suitable solutions for the XRD peaks and Checkcell software [10] is also used for indexing the peaks. According to the results obtained from the theoretical analysis by FullProf and Checkcell software, the best solutions for the two compounds showed that they both have monoclinic system but with different space groups. The best space group for 1 and 2 are $\mathrm{P} 2_{1} / \mathrm{a}$ and $\mathrm{P} 2_{1}$, respectively. The lattice parameters for 1 and 2 are presented in Table 1. Also the calculated miller indices for 1 and 2 are tabulated in Tables 2(a,b), respectively. The average crystallite size, $\mathrm{L}$, can be calculated by using the following Scherrer's 
equation [11]:

$$
L=\frac{0.98 \lambda}{y \cos \theta},
$$

where $\gamma$ is the width at the half-maximum peak

Table 1: Lattice parameters of Chloroquinoxaline derivatives.

\begin{tabular}{|c|c|c|c|c|c|c|c|}
\hline Compound & $\mathrm{a}(\AA)$ & $\mathrm{b}(\AA)$ & $c(\AA)$ & $\alpha^{\circ}$ & $\beta^{\circ}$ & $\gamma^{\circ}$ & $\begin{array}{l}\text { Crystal system/ } \\
\text { Space group }\end{array}$ \\
\hline 1 & $\begin{array}{l}21.4108 \\
\pm 0.0152\end{array}$ & $\begin{array}{l}12.6393 \\
\pm 0.0058\end{array}$ & $\begin{array}{l}7.4320 \\
\pm 0.0058\end{array}$ & 90 & $\begin{array}{l}97.67 \\
\pm 0.142\end{array}$ & 90 & $\begin{array}{l}\text { Monoclinic/ } \\
\mathrm{P}_{2} 1\end{array}$ \\
\hline 2 & $\begin{array}{l}20.8532 \\
\pm 0.0282 \\
\end{array}$ & $\begin{array}{l}6.7970 \\
\pm 0.0043 \\
\end{array}$ & $\begin{array}{l}16.7048 \\
\pm 0.0243 \\
\end{array}$ & 90 & $\begin{array}{l}124.26 \\
\pm 0.076\end{array}$ & 90 & $\begin{array}{l}\text { Monoclinic/ } \\
\mathrm{P}_{2} 1 / \mathrm{a}\end{array}$ \\
\hline
\end{tabular}

Table 2 (a): Miller indices of derivative 1.

\begin{tabular}{lllll}
\hline $\begin{array}{c}\text { Peak } \\
\text { No. }\end{array}$ & \multicolumn{1}{c}{$2 \theta_{\text {obs }}$} & $2 \theta_{\text {cal. }}$ & \multicolumn{1}{c}{$\Delta(2 \theta)$} & h k 1 \\
\hline \hline 1 & 8.1304 & 8.1304 & -0.0052 & $\overline{1} 10$ \\
2 & 10.8807 & 10.8807 & 0.002 & 210 \\
3 & 11.9993 & 11.9993 & -0.0068 & 001 \\
4 & 13.9986 & 13.9986 & -0.0037 & 020 \\
5 & 14.6174 & 14.6174 & 0.004 & $\overline{1}_{20} 20$ \\
6 & 16.3356 & 16.3356 & 0.023 & $\underline{2} 20$ \\
7 & 18.8485 & 18.8485 & 0.0336 & $\overline{3} 20$ \\
8 & 19.6064 & 19.6064 & -0.0169 & $\overline{2} 21$ \\
9 & 21.4712 & 21.4712 & -0.0147 & $\overline{1} 30$ \\
10 & 22.7085 & 22.7085 & 0.0178 & 230 \\
11 & 24.3293 & 24.3293 & 0.0106 & 031 \\
12 & 25.1532 & 25.1532 & -0.0077 & 600 \\
13 & 26.0931 & 26.0931 & 0.0074 & 421 \\
14 & 26.6536 & 26.6536 & -0.0289 & $\overline{3} 12$ \\
15 & 27.9977 & 27.9977 & -0.0218 & 022 \\
16 & 28.8849 & 28.8849 & -0.0224 & $\overline{6} 20$ \\
17 & 30.9672 & 30.9672 & -0.0043 & $\overline{3} 40$ \\
18 & 32.21 & 32.21 & -0.0489 & 032 \\
19 & 36.6062 & 36.6062 & 0.0361 & 003 \\
20 & 42.5577 & 42.5577 & -0.0136 & 100 \\
\hline \hline
\end{tabular}

Table 2 (b): Miller indices of derivative 2.

\begin{tabular}{|c|c|c|c|c|}
\hline $\begin{array}{c}\text { Peak } \\
\text { No. } \\
\end{array}$ & $2 \theta \mathrm{obs}$ & $2 \theta \mathrm{cal}$. & $\Delta(2 \theta)$ & h k 1 \\
\hline 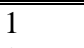 & 6.3844 & 6.3967 & -0.0123 & 4001 \\
\hline 2 & 10.7821 & 10.7862 & -0.0041 & 102 \\
\hline 3 & 12.9161 & 12.925 & -0.0089 & $\overline{3} 01$ \\
\hline 4 & 14.1336 & 14.1388 & -0.0052 & $\overline{1} 11$ \\
\hline 5 & 17.0603 & 17.0408 & 0.0195 & $\overline{4} 02$ \\
\hline 6 & 20.1449 & 20.2226 & -0.0777 & $\overline{3} 10$ \\
\hline 7 & 22.8218 & 22.8181 & 0.0037 & $\overline{4} 13$ \\
\hline 8 & 24.3711 & 24.3604 & 0.0107 & 212 \\
\hline 9 & 25.3653 & 25.3591 & 0.0062 & $\overline{2} 14$ \\
\hline 10 & 26.0066 & 26.0181 & -0.0115 & $\overline{6} 02$ \\
\hline 11 & 27.0103 & 26.9977 & 0.0126 & 021 \\
\hline 12 & 28.38 & 28.3861 & -0.0061 & 312 \\
\hline 13 & 39.5161 & 39.5234 & -0.0073 & 314 \\
\hline 14 & 44.4739 & 44.4786 & -0.0047 & 621 \\
\hline
\end{tabular}

intensity, measured in radians, $\lambda$ is the $\mathrm{X}$-ray wavelength and $\boldsymbol{\theta}$ is the Bragg's angle.). the values of the average crystallite size for 1 and 2 are calculated and found to be $35.5 \mathrm{~nm}$ and 25.6 $\mathrm{nm}$, respectively.

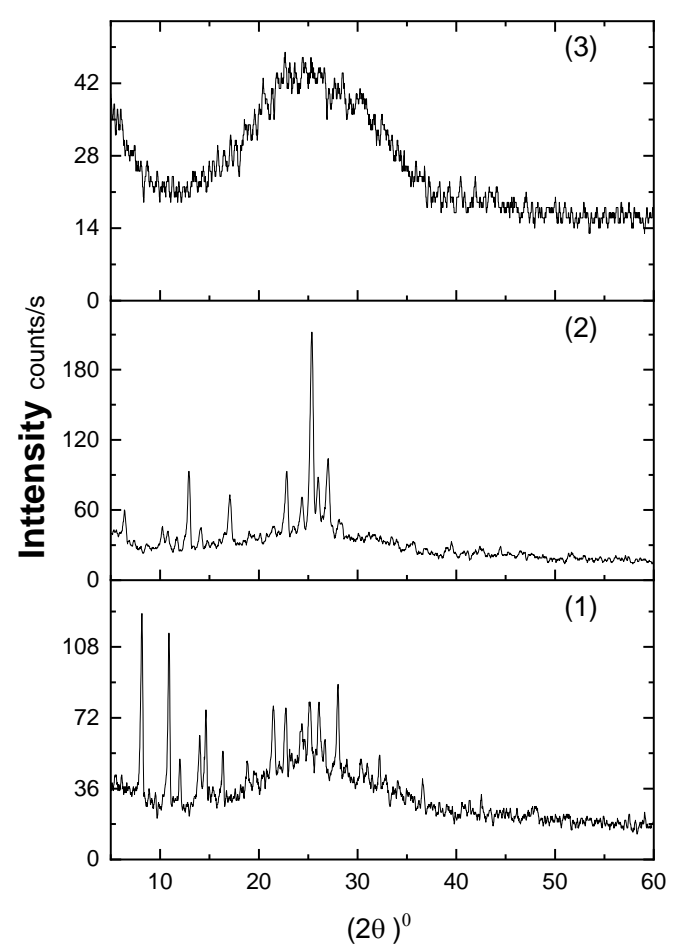

Fig. 2: X-ray diffraction pattern (XRD) for the compound 1,2 and 3 in powder from.

\section{Differential scanning calorimetric (DSC) analysis}

The DSC charts for compound 1,2 and 3 are presented in Figs.3 (a,b and c) respectively. Fig. 3a demonstrates a single exothermic peak at 496 $\mathrm{K}$. This result confirms that compound 1 is thermally and chemically stable up to $\approx 473 \mathrm{~K}$ and starts to decompose at higher temperature. In compound 2 (Fig.3b), the DSC chart demonstrates one endothermic peak at $\approx 525 \mathrm{~K}$ which may correspond to melting process and exothermic peaks at 538,556 , and $583 \mathrm{~K}$ which may be appeared due to multi stages decomposition process. Also, compound 3 (Fig.3c), shows the same thermal and chemical stability as compound 1 but to higher 
temperature $(\approx 488 \mathrm{~K})$ while an endothermic peak appeared at $\approx 517 \mathrm{~K}$ corresponding to decomposition.

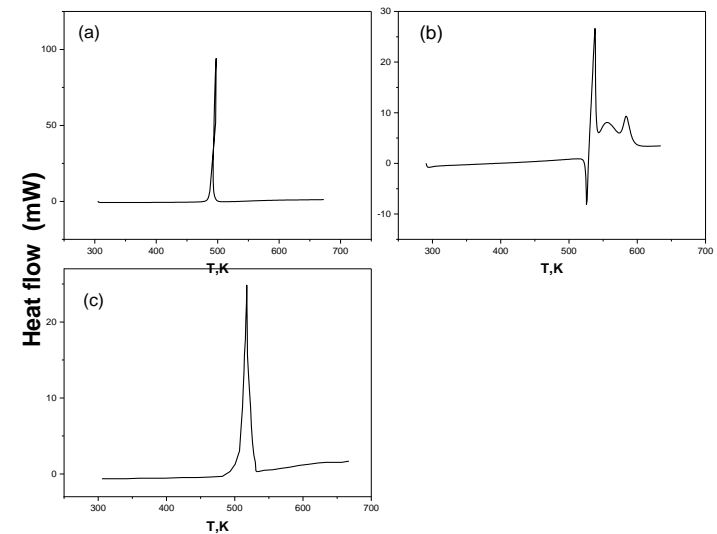

Fig. 3: DSC thermo graphs for compound 1 (a), compound 2 (b) and compound 3 (c).

\section{AC electrical properties}

Dielectric functions relate the electron transitions between energy bands of a solid to its structure; hence we may obtain useful information from the dielectric spectrum on the band structure of a solid. The dielectric constant is an important element in the design of capacitors, and knowing the properties of materials that can create capacitance. Smaller capacitors can also be manufactured using materials with a large dielectric constant, as is the case in electronics and printed circuit boards. The complex dielectric constant $\left(\varepsilon^{*}\right)$ can be determined from the measured values of the capacitance measured in parallel mode $(\mathrm{Cp})$, and loss tangent $(\tan \delta)$. The measured values of $\mathrm{C}_{\mathrm{p}}$ and $\tan \delta$ for three materials under investigation are presented in Figs. 4 and 5, respectively. The complex dielectric constant can be expressed as [12]:

$$
\varepsilon^{*}=\varepsilon_{r}+i \varepsilon_{i},
$$

in which its real part, $\varepsilon_{\mathrm{r}}$, and imaginary part, $\varepsilon_{\mathrm{i}}$, can be calculated by using the following relations $[13,14]$ :

$$
\begin{aligned}
& \varepsilon_{r}=\frac{C_{P} d}{\varepsilon_{o} A}, \\
& \varepsilon_{i}=\varepsilon_{r} \tan \delta,
\end{aligned}
$$

where $\varepsilon_{o}$ is the permittivity of free space, $d$ and $A$ are the thickness and the area of the samples, respectively. The calculated values of $\varepsilon_{\mathrm{r}}$ and $\varepsilon_{\mathrm{i}}$ in the temperature range $320-460 \mathrm{~K}$ and frequency range $100 \mathrm{~Hz}-5 \mathrm{MHz}$ are shown in Fig.6(a,b,c) and Fig.7(a,b,c), respectively . The real and imaginary parts of the dielectric constant for a fixed temperature are found to decrease with increasing frequency. Such behavior reflects dielectric relaxation process occur in the material.

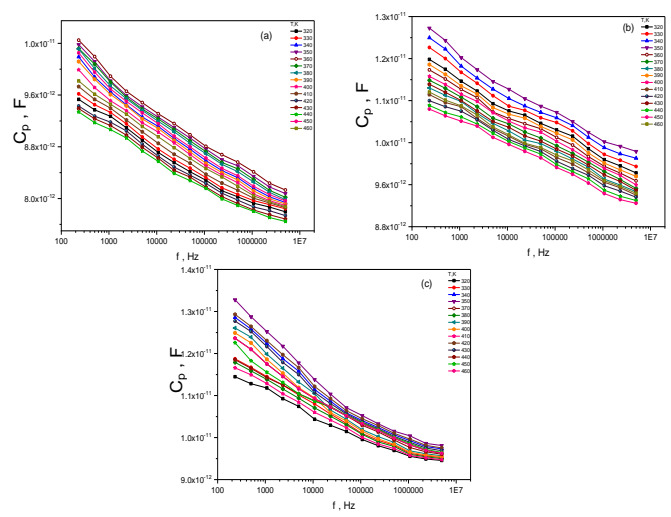

Fig. 4 (a,b,c) :the relation between the capacitance( $\mathrm{Cp}$ ) and frequency (f ) for compounds 1,2,and 3 respectively .

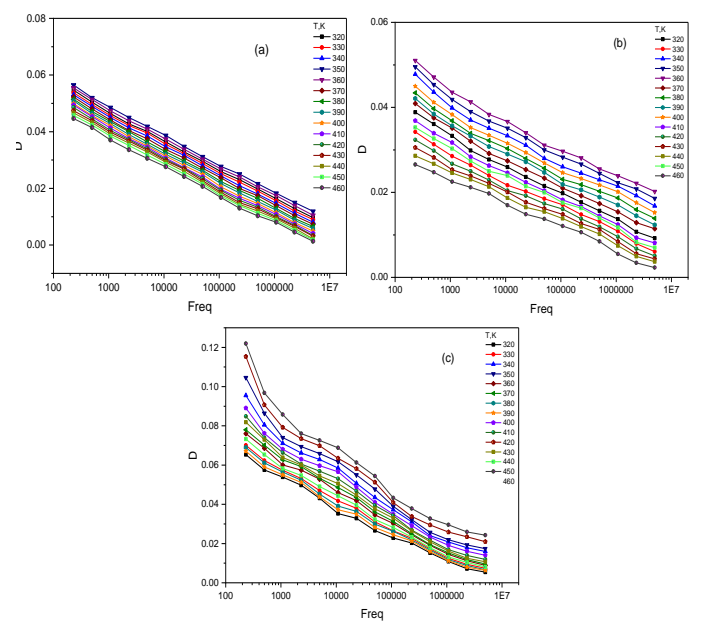

Fig. $5(\mathrm{a}, \mathrm{b}, \mathrm{c})$ :the relation between loss tangent ( tan $\sigma)$ and frequency (f ) for compounds 1,2,and respectively .

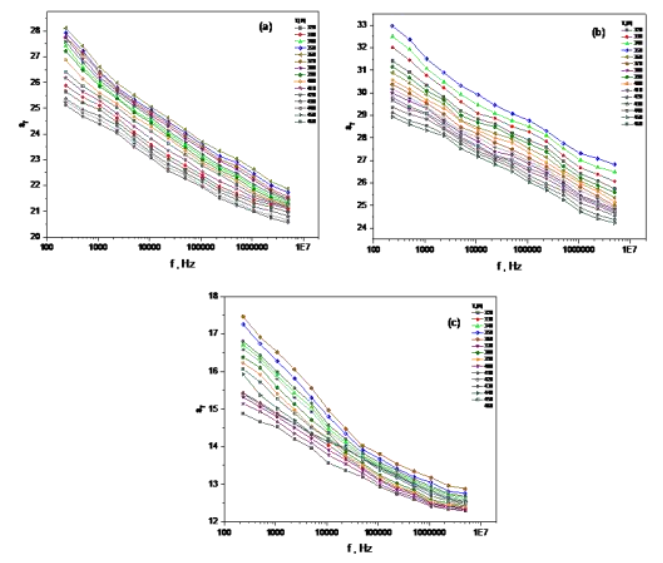

Fig. 6 (a,b,c) : Temperature and frequency dependence of the real part of the dielectric constant for compounds 1,2, and 3 respectively. 


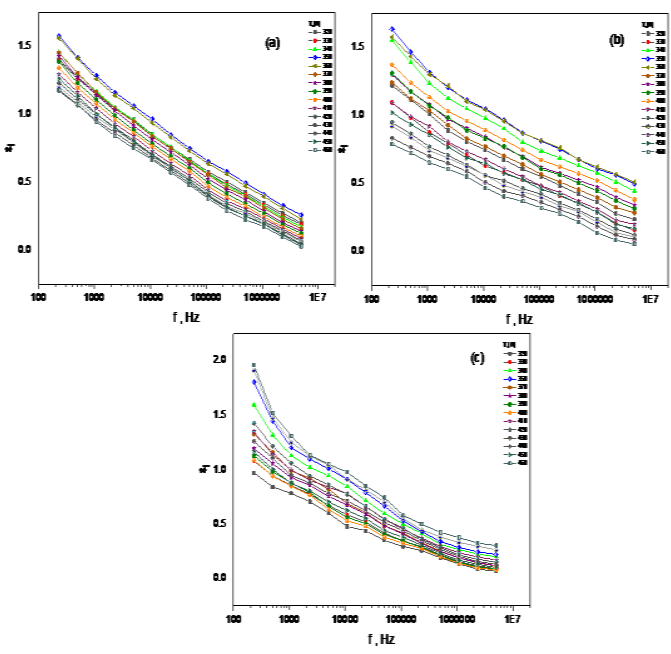

Fig. $7(a, b, c)$ : Temperature and frequency dependence of the imaginary part of the dielectric constant for compounds 1,2 , and 3 respectively.

The $\mathrm{AC}$ conductivity $\sigma_{\mathrm{ac}}$ for the compounds at a fixed temperature can be calculated by using the following relation [14]:

$$
\sigma_{\mathrm{ac}}=\omega \varepsilon_{\mathrm{i}} \varepsilon_{\mathrm{o}}
$$

where $\omega$ is the angular frequency. The AC conductivity $\sigma_{\mathrm{ac}}$ can be also expressed as [15]:

$$
\sigma_{\mathrm{ac}}=\mathrm{A}^{*} \omega^{\mathrm{s}}
$$

where $A^{*}$ is a constant, and $S$ is the power exponent that its behavior with temperature determines the type of charge carrier's conduction mechanism. The dependence of $\log$ $\sigma_{\mathrm{ac}}$ on $\log \omega$ at different temperatures for compounds 1, 2 and 3 is shown in Fig. $8(\mathrm{a}, \mathrm{b}, \mathrm{c})$. It can be understood that conduction occurs in the samples due to hopping of charge carriers as $\sigma_{\mathrm{ac}}$ is proportional to $\omega$. It is found that all three derivatives have the same behavior. We can notice that the values of $\sigma_{\mathrm{ac}}$ increase with increasing the frequency. This increase of $\sigma_{\mathrm{ac}}$ can be attributed to the increase of the oscillation of electric field accompanied with increasing frequency which will lead to an increase in the polarization in compounds 1,2 and 3 in the form of conductivity increase.

The value of $\mathrm{S}$ is directly the slope of the $\log \sigma_{\mathrm{ac}}$ versus $\log \omega$ curves at higher frequency region for each fixed temperature. The temperature dependence the exponent $\mathrm{S}$ for compound 1,2 and 3 is shown in Fig.9. The value of $S$ is almost constant with increasing the temperature. This behavior recommends the Quantum Mechanical Tunneling (QMT) mechanism to be the dominant mechanism for AC conduction. This model assumes that the carrier motion occurs through quantum mechanical tunneling between localized (defect) states near the Fermi level. For the QMT mechanism, the real part of the ac conductivity and the exponent $\mathrm{S}$ are given by:

$$
\begin{gathered}
\sigma_{\mathrm{ac}}(\omega)=\mathrm{C}^{\prime} \mathrm{e}^{2} \mathrm{k}_{\mathrm{B}} \mathrm{T}\left(\alpha^{\prime}\right)^{-1}\left[\mathrm{~N}\left(\mathrm{E}_{\mathrm{F})}\right]^{2} \omega \mathrm{R}^{4}{ }_{\omega},\right. \\
\mathrm{S}=1-\left[4 / \mathrm{In}\left(1 / \omega \tau_{0}\right)\right],
\end{gathered}
$$

where $\mathrm{N}\left(\mathrm{E}_{\mathrm{F}}\right)$ is the density of states at the Fermi level $\left(\mathrm{cm}^{-3} \mathrm{eV}^{-1}\right),\left(\alpha^{\prime}\right)^{-1}$ is the spatial decay parameter of the localized wave function, $\tau_{0}$ is the characteristic relaxation time, $k_{B}$ is Boltzmann's constant, $\mathrm{T}$, is the absolute temperature and $\mathrm{C}^{\prime}$ is a numerical constant for which different values have been obtained by various authors, but can be taken as $\pi / 24$ [15]. The above results are obtained in a wide band limit, i.e for $\Delta_{0} \gg \mathrm{k}_{\mathrm{B}} \mathrm{T}$, where $\Delta_{0}$ is the bandwidth. Thus for QMT model the frequency exponent, $\mathrm{S}$, is temperature independent but frequency dependent.

\section{Bulk Modulus}

The complex Bulk modulus $\mathrm{M}^{*}$ is defined as the reciprocal of the complex dielectric permittivity according to the following relation [16]:

$$
M^{*}=\frac{1}{\varepsilon^{*}(\omega)}=M_{r}+i M_{i},
$$

where $\mathrm{M}_{\mathrm{r}}$ and $\mathrm{M}_{\mathrm{i}}$ are the real and imaginary parts of the electrical modulus, respectively. Both of $\mathrm{M}_{\mathrm{r}}$ and $\mathrm{M}_{\mathrm{i}}$ can be calculated by use of expressions below [18]:

$$
\begin{aligned}
\mathrm{M}_{\mathrm{r}} & =\frac{\boldsymbol{E}_{r}}{\left(\boldsymbol{E}_{r}\right)^{2}+\left(\boldsymbol{\varepsilon}_{i}\right)^{2}}, \\
\mathrm{M}_{\mathrm{i}} & =\frac{\boldsymbol{E}_{i}}{\left(\boldsymbol{\varepsilon}_{r}\right)^{2}+\left(\boldsymbol{\varepsilon}_{i}\right)^{2}} .
\end{aligned}
$$

The behaviors of both $\mathrm{M}_{\mathrm{r}}$ and $\mathrm{M}_{\mathrm{i}}$ as a function of angular frequency, at different selected temperatures, are depicted in Fig.10(a,b,c) and Fig.11(a,b,c), respectively. $\mathrm{M}_{\mathrm{r}}$ is found to increase with increasing frequency, which become more effective in depletion and accumulation regions. On the other hand, $\mathrm{M}_{\mathrm{i}}$ decreases with increasing angular frequency. 

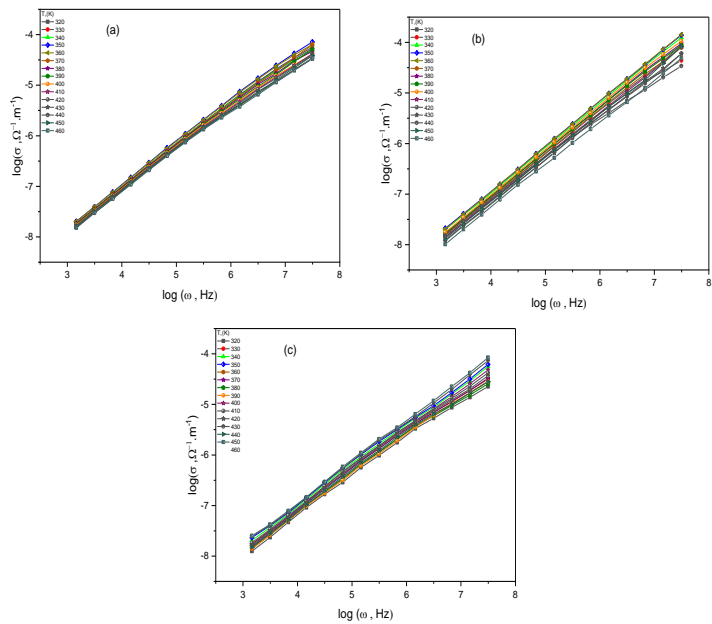

Fig. $8(a, b, c)$ :the relation between $\log \sigma a c$ and $\log \omega$ for compounds 1, 2 and 3 , respectively.

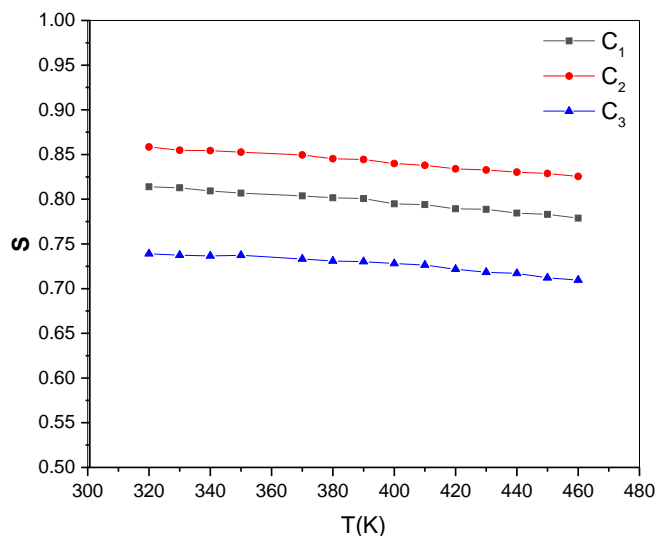

Fig. 9: The dependence of $S$ on temperature for compounds 1, 2 and 3.

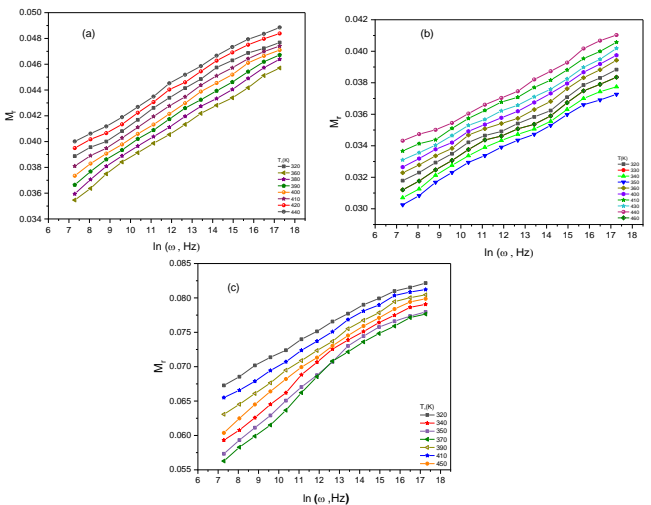

Fig.10 (a,b,c): Real part of the electric modulus, $M_{r}$ as a function of frequency for various temperatures for compounds 1,2 and 3, respectively.

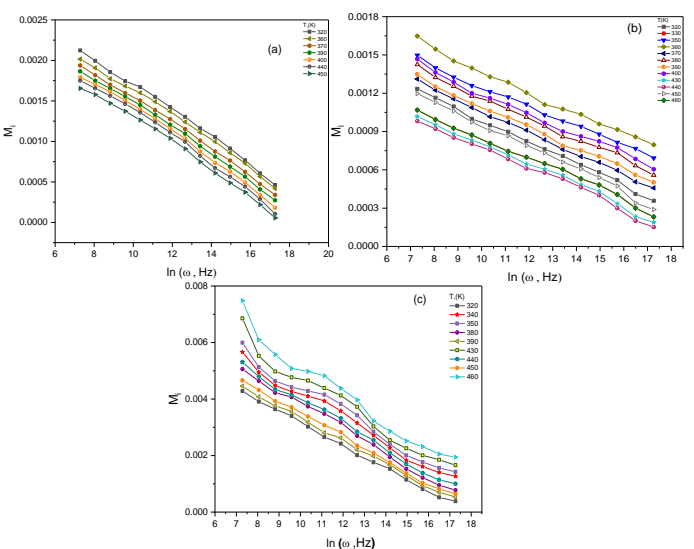

Fig.11 (a,b,c): Imaginary part of the electric modulus, $\mathrm{M}_{\mathrm{i}}$ as a function of frequency for various temperatures for compounds 1,2 and 3, respectively.

\section{Conclusion}

Structural, thermal and electrical investigations were performed on three new Chloroquinoxaline derivatives. XRD results of powder form of compounds 1 and 2 demonstrated polycrystalline and amorphous phases. Also, compound 3 had a completely amorphous phase. According to the results the best solutions for the two compounds showed that they both have monoclinic system but with different space groups and lattice parameters. DSC analyses showed that the chemical stability range differs from one derivative to another. Compound $\mathbf{1}$ is stable up to $473 \mathrm{~K}$, compound 2 up to $500 \mathrm{~K}$, and compound $\mathbf{3}$ up to $488 \mathrm{~K}$. Real and imaginary parts of the dielectric constant for a fixed temperature are found to decrease with increasing frequency. Such behavior reflected dielectric relaxation process occur in the material. AC conductivity $\sigma_{\mathrm{ac}}$ for all three derivatives increase with increasing the frequency. The effect of temperature on the ac conductivity or dielectric properties doesn't follow certain behavior and the change in the values is not much considerable. The value of $S$ for three compounds was almost constant with temperature which recommended that Quantum Mechanical Tunneling (QMT) mechanism was the dominant mechanism for AC conduction. The values of the real and imaginary parts of bulk modulus was estimated and it was found that $\mathrm{M}_{\mathrm{r}}$ increased with increasing frequency while $\mathrm{M}_{\mathrm{i}}$ decreases with increasing angular frequency. 


\section{References}

Farges, Jean-Pierre, ed. Organic conductors: fundamentals and applications. Marcel Dekker, 1994.

Brütting, Wolfgang. "Introduction to the physics of organic semiconductors." Physics of organic semiconductors (2005): 1-14.

Cheng, Guyue, et al. "Quinoxaline 1, 4-di-N-oxides: biological activities and mechanisms of actions." Frontiers in pharmacology 7 (2016): 64.

Gąsiorski, Paweł, et al. "Efficient green electroluminescence from 1, 3-diphenyl-1Hpyrazolo [3, 4-b] quinoxaline dyes in dye-doped polymer based electroluminescent devices." Dyes and Pigments 151 (2018): 380384.

$\mathrm{Wu}$, Zhonglian, et al. "Steady Enhancement in Photovoltaic Properties of Fluorine Functionalized Quinoxaline-Based Narrow Bandgap Polymer." Molecules 24.1 (2019): 54.

Hadigheh-Rezvan, Vahideh, and Bahareh PilevarMaleki. "Structural and Optical Properties of Some 5, 8-Diaminoquinoxaline Schiff Bases: Quantum Chemical Calculations." Der Chemica Sinica 9.1 (2018): 544-554.

Lizarraga, Elena, Camino Zabaleta, and Juan A. Palop. "Thermal behavior of quinoxaline 1, 4-diN-oxide derivatives." Journal of Thermal Analysis and Calorimetry" 127.2 (2017): 16551661.

Said, Samy B., Fawzia Z. El-Ablack, and Hani M. Elbeheiry. "Synthesis and Characterization of Newly Fused 1, 2-Dihydropyrido [3, 4-b], Bridged Oxadiazol-2-yl, 4-Substitutedbenzylidene Hydrazide and Arylidene 6Chloroquinoxaline 1, 4-Dioxides." Journal of the Brazilian Chemical Society 29.10 (2018): 2060-
2071.

Roisnel, Th, and Juan Rodríquez-Carvajal. "WinPLOTR: a windows tool for powder diffraction pattern analysis." Materials Science Forum. Vol. 378. No. 1. Transtec Publications; 1999, 2001.

Laugier, J., and B. CHECKCELL Bochu. "LMGP Suite of Programs for the Interpretation of X-ray Experiments, Ensp/Laboratoire des Materiaux et du Genie, Physique, Saint Martin D'heres, France, 2004.

C. Hammond "The Basics of Crystallography and Diffraction (International Union of Crystallography Texts on Crystallography) $4^{\text {th }}$ edition, Oxford University Press (2015),

Lee, Kwang-Su, Toh-Ming Lu, and X-C. Zhang. "The measurement of the dielectric and optical properties of nano thin films by $\mathrm{THz}$ differential time-domain spectroscopy." Microelectronics Journal 34.1 (2003): 63-69.

Sankarappa, T., et al. "AC conductivity and dielectric studies in $\mathrm{V} 2 \mathrm{O} 5-\mathrm{TeO} 2$ and $\mathrm{V} 2 \mathrm{O} 5-$ $\mathrm{CoO}-\mathrm{TeO} 2$ glasses." Journal of Molecular structure 889.1-3 (2008): 308-315.

Hao, Su-E., Liang Sun, and Jin-Xiang Huang. "Preparation and dielectric properties of Dy, Erdoped $\quad \mathrm{BaZr}_{0.2} \mathrm{Ti}_{0.8} \mathrm{O}_{3}$ ceramics", Materials chemistry and Physics 109 (1) (2008) 45-49.

Pollak, M., and G. E. Pike. "AC conductivity of glasses." Physical Review Letters 28.22 (1972): 1449.

Jonscher, Andrew K. "Dielectric relaxation in solids." Journal of Physics D: Applied Physics 32.14 (1999): R57.

Ambrus J. H., Moynihan C. T., and Macedo P. B. "Conductivity relaxation in a concentrated aqueous electrolyte solution." The Journal of Physical Chemistry 76.22 (1972): 3287-3295.
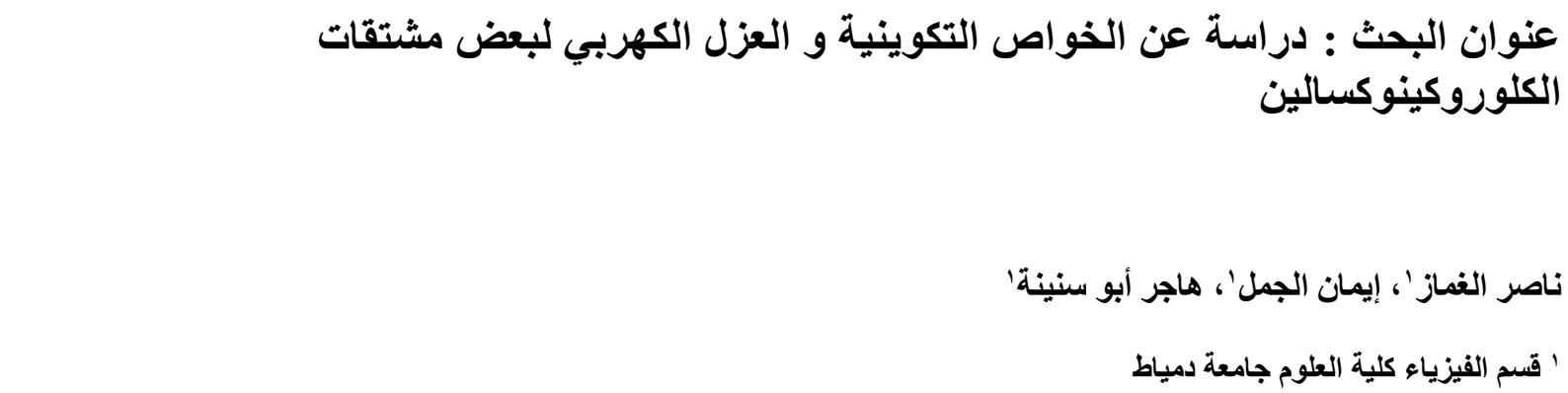

تم التحقى من الخواص التكوينية، الحرارية والكهريبة لثلاثة من مشتقات الكلوروكينوكسالين عن طريق حيود أشعة اكس 


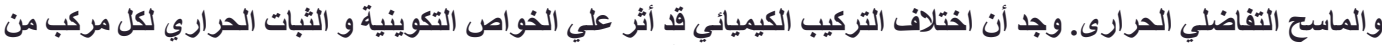

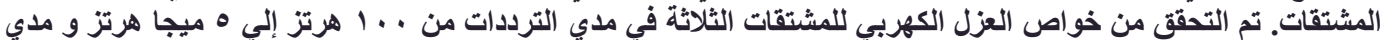

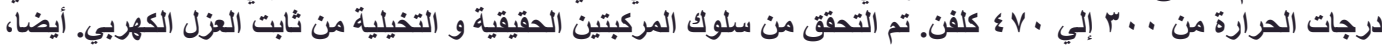

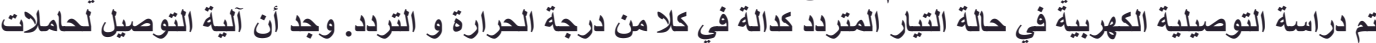

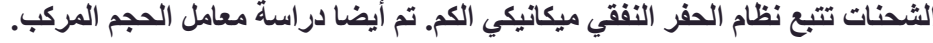

